\title{
Complete lingual palsy from bilateral Dejerine syndrome (bilateral medial medullary stroke)
}

白

Figure Imaging

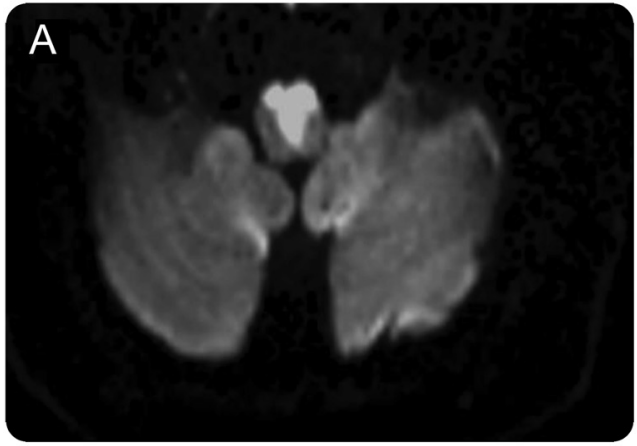

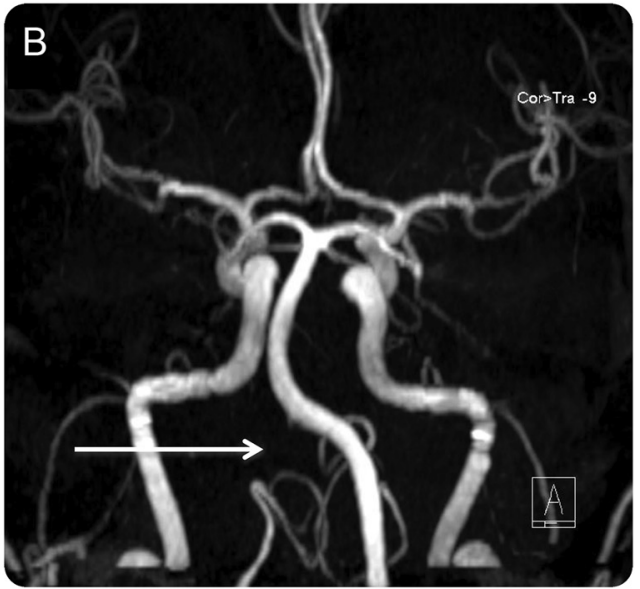

(A) Diffusion-weighted-axial image: bilateral medial medullary infarct. (B) Magnetic resonance angiography: right V4 occlusion (white arrow).

A 63-year-old man with several vascular risk factors presented with sudden left hemiplegia rapidly evolving to quadriparesis, dysarthria, bilateral hypoglossal palsy (video 1 on the Neurology ${ }^{\circledR}$ Web site at Neurology.org), and respiratory failure. IV thrombolysis with recombinant tissue plasminogen activator was administered. MRI confirmed bilateral medial medullary infarction (figure, A), and CT and magnetic resonance angiography showed right vertebral artery V4 occlusion, presumably atherosclerotic (figure, B). Tongue mobility recovered completely after 3 months (video 2).

Dejerine syndrome is a rare stroke syndrome; its bilateral form at the rostral level produces the characteristic "heart sign" on MRI ${ }^{1,2}$ (figure, A).

Spyridoula Tsetsou, MD, Isabelle Beuchat, MD, Inês Laginha, MD, PhD, Patrik Michel, MD

From Centre Hospitalier Universitaire Vaudois (S.T., I.B., P.M.), University Hospital Lausanne; and Swiss Paraplegic Center (I.L.), Nottwil, Switzerland.

Author contributions: Dr. Tsetsou: drafting the manuscript, study concept, supervision and coordination. Dr. Beuchat: drafting the manuscript, literature search, video collection. Dr. Laginha: video collection. Dr. Michel: study supervision, corrections of the manuscript.

Study funding: No targeted funding reported.

Disclosure: The authors report no disclosures relevant to the manuscript. Go to Neurology.org for full disclosures.

Correspondence to Dr. Tsetsou: stsetsou@hotmail.com

1. Pongmoragot J, Parthasarathy S, Selchen D, Saposnik G. Bilateral medial medullary infarction: a systematic review. J Stroke Cerebrovasc Dis 2013;22:775-780.

2. Tokuoka K, Yuasa N, Ishikawa T, et al. A case of bilateral medial medullary infarction presenting with "heart appearance" sign. Tokai J Exp Clin Med 2007;32:99-102. 


\section{Neurology}

\section{Complete lingual palsy from bilateral Dejerine syndrome (bilateral medial medullary stroke)}

Spyridoula Tsetsou, Isabelle Beuchat, Inês Laginha, et al.

Neurology 2016;87;550

DOI 10.1212/WNL.0000000000002926

\section{This information is current as of August 1, 2016}

\section{Updated Information \& Services}

Supplementary Material

\section{References}

Subspecialty Collections

Permissions \& Licensing

Reprints including high resolution figures, can be found at: http://n.neurology.org/content/87/5/550.full

Supplementary material can be found at: http://n.neurology.org/content/suppl/2016/07/30/WNL.0000000000002 926.DC1

This article cites 2 articles, 0 of which you can access for free at: http://n.neurology.org/content/87/5/550.full\#ref-list-1

This article, along with others on similar topics, appears in the following collection(s):

Clinical neurology examination

http://n.neurology.org/cgi/collection/clinical_neurology_examination MRI

http://n.neurology.org/cgi/collection/mri

Other cerebrovascular disease/ Stroke

http://n.neurology.org/cgi/collection/other_cerebrovascular_disease_st roke

\section{Other Education}

http://n.neurology.org/cgi/collection/other_education

Information about reproducing this article in parts (figures,tables) or in its entirety can be found online at:

http://www.neurology.org/about/about_the_journal\#permissions

Information about ordering reprints can be found online:

http://n.neurology.org/subscribers/advertise

Neurology ${ }^{\circledR}$ is the official journal of the American Academy of Neurology. Published continuously since 1951, it is now a weekly with 48 issues per year. Copyright @ 2016 American Academy of Neurology. All rights reserved. Print ISSN: 0028-3878. Online ISSN: 1526-632X.

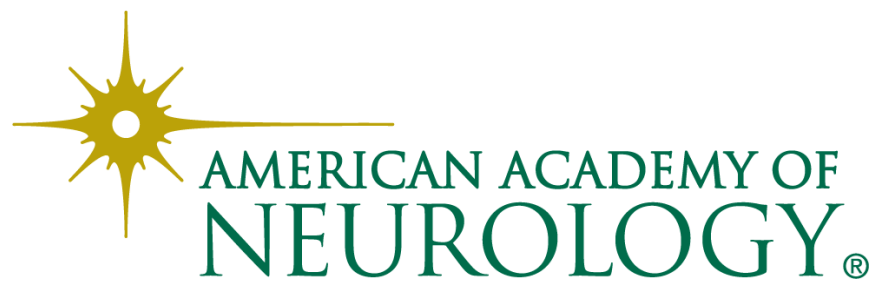

